\title{
Electron's Orbit Is Due to Some Opposite Charge Repulsion
}

\author{
Akande Raphael Oluwole
}

\begin{abstract}
We do not dispute the fact that like charges repel and opposite charges attract. However, we want to probe, theoretically, the conditions under which such attraction or repulsion takes place and to what extent does photon aid the process. Here, in this paper, we provide a work through from the interactions between the electron and protons leading to orbits of electrons. Our assumption is that, since photon is a packet of energy, we have assumed that each packet of quantized energy is converted to a ring, which we assume to be the unit packet of a phonon. In essence, we assume a phonon to be a spring made from the combination of growing rings converted from photon's packet of energy. Subsequent conversion from photon to phonon results in a compressed spring ready to be relaxed. Hence, we provide a new concept about the well known photon-phonon interaction and such that the interaction is conservative. At the same time, this paper explains same charge attraction and opposite charge repulsion.
\end{abstract}

Index Terms-Electron, nucleus, protons, photon, phonon, charge, orbit.

\section{INTRODUCTION}

Theories and researches about the formation of atoms are many and varied. This work provides a rather new concept about the formation of atoms. This paper re-considers the works of Rutherford that the electron orbiting the nucleus would spiral inwards, to the modifications by Bohr preventing it from collapsing into the nucleus, as both correlating. According to the work of [1], we know the electron carries angular momentum. Also the repulsion of oppositely charged macro ions, as reported in [2], shows that opposite charged bodies might also repel themselves. Some forms of twisted photons were also reported by [3] which are some structures of photons. These are the basic points that show up in this work.

\section{Methodology}

\section{A. Charged Energetic Particles}

Photon is the mediating particle between charged bodies [4]-[9]. In this paper, we have modeled a form of conversion from photon to phonon [6]. Since photon is a packet of energy, we have assumed that each packet of quantized energy is converted to a ring, which we assume to be the unit packet of a phonon. In essence, we assume a phonon to be a spring made from the growing of rings as they are converted from photon's packets of energy. Subsequently, the

Manuscript received May 15, 2014; revised July 15, 2014.

Akande Raphael Oluwole is with Theoretical Physics Group, University of Ibadan, Nigeria (e-mail: raphaelakande@myashiritycode.com). continuous conversion from photon to phonon results in a compressed spring ready to be relaxed. More importantly, we know that at any instance of time and at any point in space, an energetic particle is always a center of attraction for less energetic particles. Focusing on an atom, we have that the energetic particle is the nucleus and the attracted are its electrons. Like other works, we shall consider the simplest case which is the hydrogen atom. To start with, we shall define a unit distance called $\mathrm{x}$-unit which is the quantized distance expected to be covered by the attracted particle.

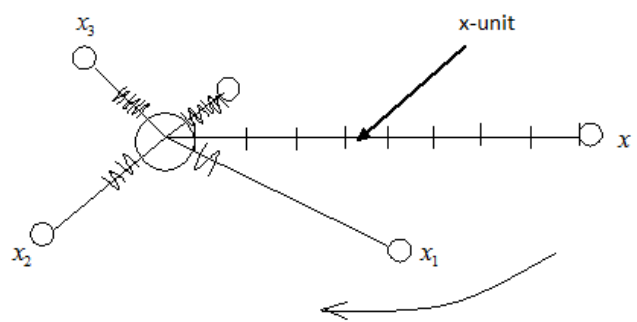

Fig. 1. The making of an atom.

In the Fig. 1 above, the $\mathrm{x}$-unit is the step of motion of the attracted electron towards the nucleus. We have modeled the photon-phonon interaction such that at each step of $\mathrm{x}$-unit, there is creation of a unit ring of energy, phonon. The $\mathrm{x}$-unit can be given as:

$$
x=\frac{\left(E_{c}-E_{e}\right)}{\exp \left(\Lambda_{e c}\right)}
$$

where

$$
\Lambda_{e c}=\frac{1}{\left[a^{\dagger}+\sin \left(2 \pi\left(\frac{b^{\dagger}}{4}\right)\right)^{2}\right]+\left[a^{\dagger}+\cos \left(2 \pi\left(\frac{b^{\dagger}}{2}\right)\right)^{2}\right]}
$$

where $a^{\dagger}$ and $b^{\dagger}$ are the operators for checking the charge and spin of two particles respectively. For application, we shall make use of something like $e^{-\Lambda_{i c}} . E_{c}$ is the energy of the nucleus, and $E_{e}$ is the energy of each of the electron being attracted. Should we have a none nucleus-electron interaction, we provide in Table I below some constants to depict the strength of the interactions based on the charge type and spin orientations.

For the above equation (1b), we can have this chart.

\section{B. Phonon Creation and Actions}

Once each packet of energy in the photon gets converted to a ring, the rings start 1) combining to each other or grow and 2 ) as the spring is formed, each ring spin flips the attracted 
electron.

For the simplest of cases, we can express the creation of phonon by photon is given as:

$$
p h t \propto p h n
$$

We have defined

$$
p h t=\sigma x^{2}+\sigma g x
$$

and

$$
p h n=g \sin (\sigma x-q x+\varphi)
$$

where $g=e^{-\Lambda}, \sigma$ is the instantaneous energy of the photon, $\varphi$ is the slight delay between the conversion from one form to another and $q$ is a resistance due to the type of spring considered. Fig. 2 is the photon peg which pulls the particles together irrespective of their charge. Fig. 3 (a) and Fig. 3 (b)

\begin{tabular}{|c|c|c|c|}
\hline Symbol & Value & $a^{\dagger}$ & $b^{\dagger}$ \\
\hline$\Lambda_{\uparrow \uparrow \text { or }}^{++} \Lambda_{\downarrow \downarrow}^{++}$ & 1 & 0 & 0 \\
\hline$\Lambda_{\uparrow \uparrow \text { or }}^{--} \Lambda_{\downarrow \downarrow}^{--}$ & 1 & 0 & 0 \\
\hline & $\frac{1}{2}$ & 0 & 1 \\
\hline & $\frac{1}{2}$ & 0 & 1 \\
\hline$\Lambda_{\uparrow \downarrow}^{+-}$ & $\frac{1}{4}$ & 1 & 1 \\
\hline$\Lambda_{\downarrow \downarrow \text { or }}^{+-} \Lambda_{\uparrow \uparrow}^{+-}$ & $\frac{1}{3}$ & 1 & 0 \\
\hline
\end{tabular}
are the two spring types considered which are for same charge attraction and opposite charge attraction respectively.

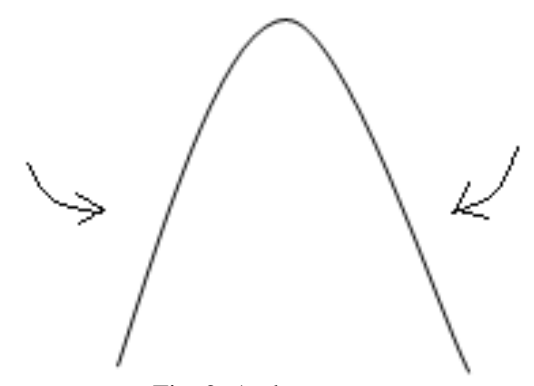

Fig. 2. A photon peg.

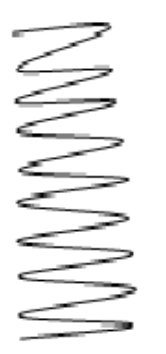

(a)

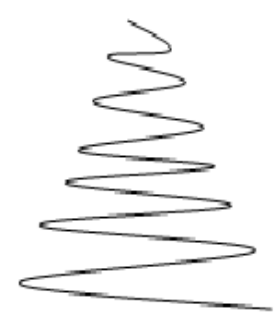

(b)
Fig. 3. Phonon Springs due to repulsion and attraction respectively.
But pht $=\vec{\varepsilon} p h n$, where $\vec{\varepsilon}$ is the constant of proportionality and also the spin orientation of the rings while being created and is given as:

$$
\vec{\varepsilon}=\vec{\varepsilon}_{x}\left(\begin{array}{cc}
0 & 1 \\
1 & 0
\end{array}\right)+\vec{\varepsilon}_{y}\left(\begin{array}{cc}
0 & -i \\
i & 0
\end{array}\right)+\vec{\varepsilon}_{z}\left(\begin{array}{ll}
1 & 0 \\
0 & 1
\end{array}\right)
$$

therefore we have a quadratic equation:

$$
\sigma x^{2}+\sigma g x-\vec{\varepsilon} g \sin (\sigma x-q x+\varphi)=0
$$

But we cannot solve this with the conventional means. So we differentiate both sides of the equation twice to give:

$$
x=\frac{1}{(\sigma-q)}\left[\sin ^{-1}\left(\frac{-2 \sigma}{\vec{\varepsilon} g(\sigma-q)^{2}}\right)-\varphi\right]
$$

This is the equation which shows the relationship between the production of phonon from photon per x-unit.

\section{Spin Flips and Orbit of Electron}

As earlier mentioned, the growth of the phonons automatically spin flips the electron. As this is done, it also provides an extra angular momentum by which the electron is proportional driven forwards alongside spin flips [6].

Firstly, we have $l=m x$, where $m$ is the number of $\mathrm{x}$-units form the first sight of electron up to where the nucleus is located at the very first instance, the beginning of attraction. However, knowing that there is an attraction means the consumption of $\mathrm{x}$-unit is nonlinear. Instead, we have: $l=-\operatorname{cosech} \bar{x}$ [7]. This now explains the shortening of $l$ per x-unit. Also, the angular displacement of the electron per $\mathrm{x}$-unit is given as: $x=-2 e^{-\Lambda} e^{-\frac{\vartheta}{2}}$, where $\xi=e^{-\Lambda}$. We shall define $\breve{x}=\frac{x}{x-1}$. With these definitions, we have $\frac{d l}{d \vartheta}=\frac{d l}{d x} \frac{d x}{d \vartheta}$, where $\frac{\partial x}{\partial \vartheta}=e^{-\Lambda} e^{-\frac{\vartheta}{2}}, \frac{\partial l}{\partial x}=\frac{1}{(x-1)^{2}} \operatorname{coth} \breve{x} \cos e c h \breve{x}$ and $\frac{d l}{d \vartheta}=\xi e^{-\frac{\vartheta}{2}}(\operatorname{coth} \breve{x})^{\delta_{i j}} \operatorname{cosech} \breve{x}$, where $\delta_{i j}=0$ for opposite charge and $\delta_{i j}=1$ for same charge.

Noting that the conservatory oscillation between the photon and phonon creates the spin flip and orbit, we have:

$$
\begin{array}{r}
m \frac{d^{2} l}{d x^{2}}+(\xi+\zeta) l=0 \propto \frac{d l}{d \vartheta} \\
m \frac{d^{2} l}{d x^{2}}-\phi^{\dagger} \phi \frac{d l}{d \vartheta}+(\xi+\zeta) l=0
\end{array}
$$

where $\phi^{\dagger} \phi$ is the constant of proportionality which we have chosen to be the in and out flow of external photons. That is 
the number of photons coming in or going out. This is because they are the ones that make the electron's instantaneous positions in its orbit uncertain and probabilistic. $\zeta$ is the net sum of other external forces acting on the attracted electron.

However, we need a term that contains the two coupled parameters: $\theta$ and $x$. So we want $\frac{d^{2} l}{d \vartheta^{2}}$ in place of $\frac{d^{2} l}{d x^{2}}$. Equation (5) above first becomes:

$$
m \frac{\partial^{2} l}{\partial x \partial \vartheta}-\phi^{\dagger} \phi \frac{d l}{d \vartheta}+(\xi+\zeta) l=0
$$

Though good, the above equation is not good enough to be easily solved, so we try to probe the relationship between $\frac{d^{2} l}{d \vartheta^{2}}$ and $\frac{\partial^{2} l}{\partial x \partial \vartheta}$ in $\frac{d^{2} l}{d \vartheta^{2}} \propto \frac{\partial^{2} l}{\partial x \partial \vartheta}$. This gives us:

$$
\frac{\left(\frac{d^{2} l}{d \vartheta^{2}}\right)}{\left(\frac{\partial^{2} l}{\partial x \partial \vartheta}\right)}=\frac{-\left(\frac{1}{4}\right)}{\frac{1}{(x-1)^{2}} \operatorname{coth} \bar{x}}=\frac{\left(\frac{d^{2} l}{d \vartheta^{2}}\right)}{\left(\frac{\partial^{2} l}{\partial x \partial \vartheta}\right)}=\frac{-\left(\frac{1}{4}\right) \operatorname{coth} \bar{x}}{\frac{1}{(x-1)^{2}}}
$$

or

$$
\frac{d^{2} l}{d \vartheta^{2}}=-\left(\frac{(x-1)^{2 \delta_{i j}}}{4}\right)\left[(\operatorname{coth} \widetilde{x})^{2 \delta_{i j}-1}\right] \frac{\partial^{2} l}{\partial x \partial \vartheta}
$$

With

$$
\frac{d^{2} l}{d \vartheta^{2}}=\frac{1}{4} \xi e^{-\frac{\vartheta}{2}} \operatorname{cosech} \widetilde{x}
$$

and

$\frac{d^{2} l}{d \vartheta^{2}}=\frac{1}{4} \xi e^{-\frac{\vartheta}{2}} \operatorname{coth} \breve{x} \operatorname{cosech} \breve{x} \quad$ for $\quad \delta_{i j}=0$ and $\delta_{i j}=1$ respectively, and $\frac{\partial^{2} l}{\partial x \partial \vartheta}=-\frac{1}{(x-1)^{2}} \xi e^{-\frac{\vartheta}{2}} \operatorname{cosech} \breve{x}$ and $\frac{\partial^{2} l}{\partial x \partial \vartheta}=-\frac{1}{(x-1)^{2}} \xi e^{-\frac{\vartheta}{2}} \operatorname{coth} \breve{x} \operatorname{cosech} \breve{x}$ for $\delta_{i j}=0$ and $\delta_{i j}=1$ respectively.

\section{RESUlts}

Now, we have (6) become:

$$
-\left(\frac{4}{(x-1)^{2 \delta_{i j}}(\operatorname{coth} \breve{x})^{2 \delta_{i j}-1}}\right) \frac{d^{2} l}{d \vartheta^{2}}-\phi^{\dagger} \phi \frac{d l}{d \vartheta}+(\xi+\zeta) l=0
$$

If we define $\omega=-\left(\frac{4}{(x-1)^{2 \delta_{i j}}(\operatorname{coth} \widetilde{x})^{2 \delta_{i j}-1}}\right)$, we have

$$
\frac{d^{2} l}{d \vartheta^{2}}-\omega_{0}^{2} \frac{d l}{d \vartheta}+\left(\omega_{1}^{2}+\omega_{2}^{2}\right) l=0
$$

where

$$
\begin{gathered}
\omega_{o}^{2}=\sqrt{-\frac{\phi^{\dagger} \phi}{m \omega}}=i \sqrt{\frac{\phi^{\dagger} \phi}{m \omega}}, \omega_{1}^{2}=\sqrt{-\frac{\xi}{m \omega}}=i \sqrt{\frac{\xi}{m \omega}} \\
\omega_{2}^{2}=\sqrt{-\frac{\zeta}{m \omega}}=i \sqrt{\frac{\zeta}{m \omega}}
\end{gathered}
$$

the solution of which is:

$$
\aleph_{ \pm}=\frac{i}{2} \sqrt{\frac{\phi^{\dagger} \phi}{m \omega}} \pm \frac{1}{2} \sqrt{\frac{-\phi^{\dagger} \phi \sqrt{m \omega}-i 4 m \omega(\xi+\xi)}{(m \omega)^{3 / 2}}}
$$

By expanding the second term by Binomial series, we have:

$$
\aleph_{ \pm}=\frac{i}{2} \sqrt{\frac{\phi^{\dagger} \phi}{m \omega}}\left\{1 \pm S_{j}\right\}
$$

where $S_{j}$ is given as:

$$
\begin{gathered}
S_{j}=1+2 i y+2 y^{2}-4 i y^{3}-10 y^{4}+3072 i y^{5}+ \\
60480 y^{6}-1330560 i y^{7}-34594560 y^{8}+\ldots \\
y=\frac{i 4 m \omega(\xi+\xi)}{\phi^{\dagger} \phi \sqrt{m \omega}}
\end{gathered}
$$

where

$$
\mathfrak{I}=\alpha e^{i \aleph_{+} \vartheta}+\beta e^{i \aleph_{-} \vartheta}
$$

The equation above describes the path, orbit, of the electron about the nucleus. Except for $\phi^{\dagger} \phi$ which is the only probable parameter, the path of the electron would have been predictable.

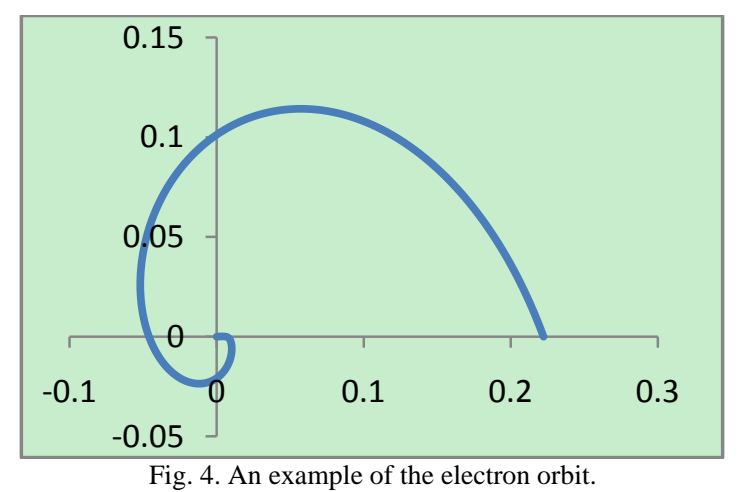

The orbit restarts along the $x$-axis or anywhere on $x$-y plane.

\section{CONCLUSION}

We have the conviction that the conversion of photon to 
phonon and vice versa produces the orbit for the electron around the nucleus. This being possible mostly because both are of opposite charge. For same charged particle, a clear cut orbit might be very difficult to create due to the type of phonon spring made, see Fig. 3. The probability in the position of electron on its orbits is due to the probabilistic inand-outwards flow of photon or phonon during the interaction.

We have shown how the ordinary photon-phonon interaction produces different types of phonon springs. With the same model, of ours, we also state the relationship between this interaction and the orbit of electron about the nucleus, all with a single model. Our model details the dynamics of the atom.

The spiral shape of the electron's orbit reveals the Rutherford's [8] thought of an electron spiralling into the nucleus. Had it not been for the creation of phonon springs, the electron would have spiralled inwards to the nucleus. So also, our model reveals the possible reason why the galaxies are in spiral shapes and could also, possibly, reveal the reason for the angular assembly of water molecules.

\section{REFERENCES}

[1] M. Uchida and A. Tonomura, "Generation of electron beams carrying orbital angular momentum," Nature, vol. 464, pp. 737-739, DOI: 10.1038 /nature 08904 .
[2] Y.-S. Jho et al., "Repulsion between oppositely charged planar macroions," DOI: 10.1371/journal.pone.0069436.

[3] M.-T. Gabriel, J. P. Torres, and L. Torner, "Twisted photons," Nature Physics, vol. 3, pp. 305-310, DOI: 10.1038/nphys607.

[4] S. N. Ghoshal, Atomic and Nuclear Physics, S. CHAND Company, India.

[5] R. H. Landau and M. Jose, Computational Physics, Wiley-VCH Verlag $\mathrm{GmbH} \& \mathrm{Co} . \mathrm{KGaA}$.

[6] P. Coleman, Introduction to Many-Body Physics, 2011.

[7] K. F. Riley, M. P. Hobson, and S. J. Bence, Mathematical Methods for Physics and Engineering, Cambridge University Press, 2002.

[8] D. Halliday, R. Resnick, and J. Walker, Fundamentals of Physics, John Wiley \& Sons, Inc, 2001.

[9] D. Tong, Concepts in Theoretical Physics, Lecture 6: Particle Physics, Cambridge University Press.

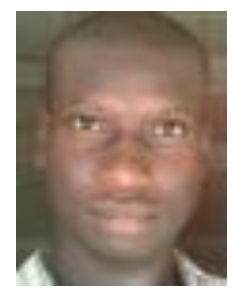

Akande Raphael Oluwole is a native of Yoruba tribe in Nigeria, West Africa. He was born in Lagos, Nigeria in November of 1982. His primary education was both in Ibadan. Also, in Ibadan, he got his BSc (2008), MSc (2011) and currently he is in the 3rd year of $\mathrm{PhD}$ at the University of Ibadan. His major field of study is in theoretical physics with special interest in modeling new materials. Oluwole speaks Yoruba and English fluently. $\mathrm{He}$ is the developer of several mathematica computer programs from chaos theory to econophysics and biophysics applications. He is vast and experienced in computational simulations with skills in C, C++, Java, Fortran, C\#, VB, Windows Shell etc. He is currently developing mobile apps, in Android and Windows Phone, for mathematical applications in all fields of science and non-sciences. Oluwole is the author of three papers in the field of spin interactions and working towards modeling the manner in which molecules acquire gravity. 